Quantum Field Theory Under the Influence

of External Conditions (QFEXT11)

International Journal of Modern Physics: Conference Series

Vol. 14 (2012) 73-87

(C) World Scientific Publishing Company

DOI: $10.1142 / \mathrm{S} 2010194512007246$

\title{
QUANTUM CORRECTIONS TO GRAVITY AND THEIR IMPLICATIONS FOR COSMOLOGY AND ASTROPHYSICS
}

\author{
JULIO C. FABRIS, PAULO L. C. DE OLIVEIRA, DAVI C. RODRIGUES \\ and ALAN M. VELASQUEZ-TORIBIO \\ Departamento de Física - CCE, \\ Universidade Federal do Espírito Santo \\ Vitória, CEP: 29060-900, ES, Brazil \\ fabris@pq.cnpq.br; \\ davirodrigues.ufes@gmail.com; \\ alan.toribio@ufes.br \\ ILYA L. SHAPIRO \\ Departamento de Física - ICE, \\ Universidade Federal de Juiz de Fora \\ Juiz de Fora, CEP: 36036-330, MG, Brazil \\ shapiro@fisica.ufjf.br
}

Published 28 July 2012

\begin{abstract}
The quantum contributions to the gravitational action are relatively easy to calculate in the higher derivative sector of the theory. However, the applications to the postinflationary cosmology and astrophysics require the corrections to the Einstein-Hilbert action and to the cosmological constant, and those we can not derive yet in a consistent and safe way. At the same time, if we assume that these quantum terms are covariant and that they have relevant magnitude, their functional form can be defined up to a single free parameter, which can be defined on the phenomenological basis. It turns out that the quantum correction may lead, in principle, to surprisingly strong and interesting effects in astrophysics and cosmology ${ }^{\mathrm{a}}$.
\end{abstract}

Keywords: Quantum effects; Galaxies rotation curves; Cosmological parameters.

\section{Introduction}

One of the subjects which attract a lot of attention recently is a possible modification of General Relativity and its implications for observational and experimental physics. Along with the long list of possible ad hoc modifications, from the traditional versions such as scalar-tensor theories and non-linear extensions of the Einstein-Hilbert action, and up to the galileons and modern versions of massive gravity, there is one which deserves, from our viewpoint, a very special attention. Independent on whether gravity should be or should not be quantized, we know that

${ }^{a}$ Based on the talk presented by I. Shapiro. 
the matter fields should. Therefore, it is reasonable to ask whether the quantum effects of matter fields are capable to produce significant effects on the astrophysical or even cosmological scale.

At quantum level the dynamics of gravity with quantum corrections is governed by the effective equations, coming from the Effective Action (EA) of vacuum $\Gamma\left[g_{\mu \nu}\right]$,

$$
e^{i \Gamma\left[g_{\mu \nu}\right]}=\int d \Phi e^{i S\left[\Phi, g_{\mu \nu}\right]}, \quad \text { where } \Phi=\{\text { matter fields }\} .
$$

In case of renormalizable theory of matter fields we can write

$$
S\left[\Phi, g_{\mu \nu}\right]=S_{v a c}\left[g_{\mu \nu}\right]+S_{m}\left[\Phi, g_{\mu \nu}\right] \quad \text { and } \quad \Gamma\left[g_{\mu \nu}\right]=S_{v a c}\left[g_{\mu \nu}\right]+\bar{\Gamma}\left[g_{\mu \nu}\right]
$$

The classical part of the vacuum action has the form

$$
S_{v a c}=S_{E H}+S_{H D}, \quad \text { where } \quad S_{E H}=-\frac{1}{16 \pi G} \int d^{4} x \sqrt{-g}(R+2 \Lambda)
$$

and $S_{H D}$ includes higher derivative terms.

$$
S_{H D}=\int d^{4} x \sqrt{-g}\left\{a_{1} C^{2}+a_{2} E+a_{3} \square R+a_{4} R^{2}\right\} .
$$

Here

$$
C^{2}(4)=R_{\mu \nu \alpha \beta}^{2}-2 R_{\alpha \beta}^{2}+1 / 3 R^{2}
$$

is the square of the Weyl tensor and

$$
E=R_{\mu \nu \alpha \beta} R^{\mu \nu \alpha \beta}-4 R_{\alpha \beta} R^{\alpha \beta}+R^{2}
$$

the integrand of the Gauss-Bonnet topological invariant.

At the astrophysical or cosmological scale the quantum corrections in the matter fields sector can not play an important role. Therefore, from the perspective given above, the main problem is to evaluate the quantum correction to the classical action of vacuum, $\bar{\Gamma}\left[g_{\mu \nu}\right]$, at least at 1 -loop.

In the case of massless conformal fields $\bar{\Gamma}\left[g_{\mu \nu}\right]$ can be obtained, e.g., by integrating conformal anomaly ${ }^{1,2}$ (see also Ref. 3 for the technical introduction and further references)

$$
\begin{aligned}
\bar{\Gamma}_{i n d} & =S_{c}\left[g_{\mu \nu}\right]+\frac{\beta_{1}}{4} \int_{x} \int_{y}\left(E-\frac{2}{3} \square R\right)_{x} G(x, y)\left(C^{2}\right)_{y} \\
& -\frac{\beta_{2}}{8} \int_{x} \int_{y}\left(E-\frac{2}{3} \square R\right)_{x} G(x, y)\left(E-\frac{2}{3} \square R\right)_{y}+\frac{3 \beta_{3}-2 \beta_{2}}{6} \int_{x} R^{2} .
\end{aligned}
$$

Here we use the notations $\int_{x}=\int d^{4} x \sqrt{g}$ and $\Delta_{x} G\left(x, x^{\prime}\right)=\delta\left(x, x^{\prime}\right)$. Furthermore

$$
\Delta=\square^{2}+2 R^{\mu \nu} \nabla_{\mu} \nabla_{\nu}-\frac{2}{3} R \square+\frac{1}{3}\left(\nabla^{\mu} R\right) \nabla_{\mu}
$$


and $S_{c}\left[g_{\mu \nu}\right]$ is an arbitrary conformal functional. The $\beta$-functions in Eq. (5) depend on the number of fields, $N_{0}, N_{1 / 2}, N_{1}$,

$$
\left(\begin{array}{c}
\beta_{1} \\
-\beta_{2} \\
\beta_{3}
\end{array}\right)=\frac{1}{360(4 \pi)^{2}}\left(\begin{array}{c}
3 N_{0}+18 N_{1 / 2}+36 N_{1} \\
N_{0}+11 N_{1 / 2}+62 N_{1} \\
2 N_{0}+12 N_{1 / 2}-36 N_{1}
\end{array}\right)
$$

There are many important applications of conformal anomaly and the EA (5) (see, e.g., Refs. 4, 5, 6), and one of the most clear ones is the Starobinsky model of inflation ${ }^{7}$. It is interesting that the EA $S_{E H}+\bar{\Gamma}_{i n d}$ is producing two dS-like solutions for the homogeneous and isotropic metric (for simplicity we consider only spatially-flat case $k=0$ ),

$$
d s^{2}=d t^{2}-a^{2}(t) d l^{2}, \quad a(t)=a_{0} \cdot \exp (H t)
$$

where ${ }^{8}$

$$
H=\frac{M_{P}}{\sqrt{-32 \pi b}}\left(1 \pm \sqrt{1+\frac{64 \pi b}{3} \frac{\Lambda}{M_{P}^{2}}}\right)^{1 / 2} .
$$

As far as $\Lambda \ll M_{P}^{2}$, we meet two very different values of $H$ (we consider $\Lambda>0$ )

$$
H_{c} \approx \sqrt{\frac{\Lambda}{3}} \quad \text { and } \quad H_{S} \approx \frac{M_{P}}{\sqrt{-16 \pi b}} .
$$

The solution with $H_{c}$ is the one of the theory without quantum corrections. The second value $H_{S}$ corresponds to the inflationary solution of Starobinsky ${ }^{7}$.

Three relevant for us observations are in order.

- First, the expression (5) is an exact EA for the conformally flat metric, including (8) as a particular case.

- Second, such an exact solution is possible only due to the very special resummation in the EA, and can not be obtained from the massless conformal fields via the usual perturbative approach ${ }^{9}$. The perturbations in curvature tensor and its covariant derivatives will always give us non-localities related to the Green functions of the quantum fermionic, scalar and vector fields, and not the ones of the universal conformal operator $\Delta$ from (6).

- Third, nothing similar to the EA (5) is possible in the case of massive fields. One could think that the effects of massive fields can not be relevant in principle at the cosmic scale, due to the decoupling phenomenon. However, such a statement can not be mathematically proved ${ }^{10}$. In fact, some direct considerations using the Green functions of massive fields indicate that the quantum effects of such fields should be negligible, but there are two flaws in such a treatment. First of all, it is essentially based on the expansion of the metric near the flat background, and (as we already pointed out here) this approach is not safe for the massive fields. For example, the conformal parametrization - based calculations give some positive and in fact reliable output for the case of light massive fields ${ }^{8,11}$. Furthermore, one can not rule out that the EA action of massive fields can be subject of a resummation similar to the one which leads to (1) in case of anomaly-induced EA. So, after all nothing 
can be ruled out completely and therefore we have a chance to meet relevant IR vacuum quantum effects. In the rest of this contribution we shall present a general view on the problem of vacuum quantum effects of massive fields and also briefly discuss their possible effects in astrophysics and cosmology. Many technical details are omitted here, but can be found by the reader in the parallel papers Refs. 12 , 13, 14 and Refs. 15, 16.

\section{Covariance arguments for massive fields}

The vacuum quantum contributions of massive fields are much more complicated and interesting, if the low-energy effects are concerned. As we have already mentioned above, one has to account for the decoupling phenomenon, however the result may be different from what one could naively expect.

Let us start from the pedagogical example of QED. In the UV limit the one-loop corrected action of photon is

$$
-\frac{e^{2}}{4} F_{\mu \nu} F^{\mu \nu}+\frac{e^{4}}{3(4 \pi)^{2}} F_{\mu \nu} \ln \left(-\frac{\square}{\mu^{2}}\right) F^{\mu \nu}
$$

and we meet a standard Minimal Subtraction $(\overline{\mathrm{MS}})$ renormalization scheme based $\beta$-function for $e(\mu)$. Then, at low energies there is a quadratic decoupling, This means, in the framework of the Renormalization Group approach, the quadratic difference between UV and IR $\beta$-function,

$$
\begin{aligned}
& \text { UV limit } p^{2} \gg m^{2} \Longrightarrow \beta_{e}^{1 U V}=\frac{4 e^{3}}{3(4 \pi)^{2}}+\mathcal{O}\left(\frac{m^{2}}{p^{2}}\right), \\
& \text { IR limit } p^{2} \ll m^{2} \Longrightarrow \beta_{e}^{1 I R}=\frac{e^{3}}{(4 \pi)^{2}} \cdot \frac{4 p^{2}}{15 m^{2}}+\mathcal{O}\left(\frac{p^{4}}{m^{4}}\right),
\end{aligned}
$$

that is the Appelquist and Carazzone decoupling theorem ${ }^{17}$.

Similar results can be obtained for gravity, e.g., for a massive scalar field we have the following UV and IR $\beta$-functions for the parameter $a_{1}$ in the action $(4)^{18,19}$ :

$$
\begin{aligned}
& \beta_{1}^{U V}=-\frac{1}{(4 \pi)^{2}} \frac{1}{120}+\mathcal{O}\left(\frac{m^{2}}{p^{2}}\right)=\beta_{1}^{\overline{M S}}+\mathcal{O}\left(\frac{m^{2}}{p^{2}}\right), \\
& \beta_{1}^{I R}=-\frac{1}{1680(4 \pi)^{2}} \cdot \frac{p^{2}}{m^{2}}+\mathcal{O}\left(\frac{p^{4}}{m^{4}}\right),
\end{aligned}
$$

This is the Appelquist and Carazzone Theorem for gravity, it implies a quadratic suppression of the running in the IR. The same rule holds also for spin- $1 / 2$ and spin-1 fields for both $C^{2}$ and $R^{2}$ terms. All these results were obtained through the momentum-subtraction scheme, in the flat-space expansion $g_{\mu \nu}=\eta_{\mu \nu}+h_{\mu \nu}$ or in an equivalent perturbative (in curvatures) heat-kernel approach. However, it is easy to see that in the momentum-subtraction scheme $\beta_{1 / G}=\beta_{\Lambda}=0$, because the $\square$-dependent form factors like the one of Eq. (11), can not be inserted into the Hilbert-Einstein and cosmological terms ${ }^{18}$. At the same time there is no problem to insert such a form factor into $C^{2}$ term,

$$
C_{\mu \nu \alpha \beta} \ln \left(-\frac{\square}{\mu^{2}}\right) C_{\mu \nu \alpha \beta} .
$$


and similarly to $R^{2}$ term, that is why we can study the running of the corresponding parameters in the momentum-subtraction scheme of renormalization.

From the consideration presented above, it becomes clear why we get an apparent $\beta_{\Lambda}=\beta_{1 / G}=0$ in the momentum-subtraction scheme. The reason is that the expansion $g_{\mu \nu}=\eta_{\mu \nu}+h_{\mu \nu}$ is not appropriate for the massive fields case. The renormalizable theory of massive fields has to include the cosmological constant term in this case, and then $\eta_{\mu \nu}$ is not a classical solution anymore. In this situation the expansion around flat space is not a legitimate procedure. Perhaps the linearized gravity approach is simply not an appropriate tool for the $\mathrm{CC}$ and Einstein terms. If we perform some other expansion, the output for the $\beta_{\Lambda}$ and $\beta_{1 / G}$ can be different, but this is out of our knowledge at the moment.

As far as the direct theoretical derivation of the quantum effects of our interest is not possible, we can look at the problem from the phenomenological side. One can simply make an assumption that some relevant quantum contributions are present, and then use the covariance arguments to find their form. Later on we will see how this approach fits also to the Appelquist and Carazzone theorem.

Consider first the cosmological term and perform a derivative expansion in the EA. The EA $\Gamma\left[g_{\mu \nu}\right]$ can not include odd terms in metric derivatives, just because it is a covariant scalar. In the cosmological setting this means there are no $\mathcal{O}(H)$ terms, and also no $\mathcal{O}\left(H^{3}\right)$ and so on ${ }^{20}$. Hence the covariance arguments give the formula

$$
\begin{aligned}
& \rho_{\Lambda}(H)=\frac{\Lambda(H)}{16 \pi G(H)}=\rho_{\Lambda}\left(H_{0}\right)+C\left(H^{2}-H_{0}^{2}\right), \\
& \text { where } \quad C=\frac{3 \nu}{8 \pi} M_{p}^{2}\left(H^{2}-H_{0}^{2}\right)
\end{aligned}
$$

and the physical sense of the constant parameter $\nu$ will de defined later on, in Eq. (19). Starting from (15) the standard covariance (conservation law) consideration leads to the relation ${ }^{21}$

$$
G(H ; \nu)=\frac{G_{0}}{1+\nu \ln \left(H^{2} / H_{0}^{2}\right)}, \quad \text { where } \quad G\left(H_{0}\right)=G_{0}=\frac{1}{M_{P}^{2}} .
$$

From the renormalization group perspective, the identification of scale $\mu \sim H$ is the most natural in the cosmological setting ${ }^{22,23 \mathrm{~b}}$. Therefore the last formulas can be generalized as

$$
\rho_{\Lambda}(\mu)=\rho_{\Lambda}\left(\mu_{0}\right)+C\left(\mu^{2}-\mu_{0}^{2}\right) . \quad G(\mu)=\frac{G_{0}}{1+\nu \ln \left(\mu^{2} / \mu_{0}^{2}\right)},
$$

where $\mu_{0}$ is the reference scale. We will discuss an identification of $\mu$ for the astrophysical case below.

Before we proceed, it is worthwhile to make a small note on the Cosmological Constant (CC) Problem ${ }^{23,25}$. The main relation, from the QFT viewpoint, is that

\footnotetext{
${ }^{b}$ See Ref. 24 for an alternative treatment.
} 
the observed density of the cosmological constant term is a sum of the two finite terms, namely of the vacuum and induced one,

$$
\rho_{\Lambda}^{o b s}=\rho_{\Lambda}^{v a c}\left(\mu_{c}\right)+\rho_{\Lambda}^{i n d}\left(\mu_{c}\right)
$$

where $\mu_{c} \propto H_{0}$ is the late Universe cosmic scale. Here $\rho_{\Lambda}^{o b s}$ is the value which is likely observed in SN-Ia, LSS, CMB etc, to be $\rho_{\Lambda}^{o b s}\left(\mu_{c}\right) \approx 0.7 \rho_{c}^{0} \propto 10^{-47} \mathrm{GeV}^{4}$. The unusual feature of the relation (18) is that the two terms $\rho_{\Lambda}^{v a c}\left(\mu_{c}\right)$ and $\rho_{\Lambda}^{\text {ind }}\left(\mu_{c}\right)$ in the r.h.s. are evaluated at the (at least) Fermi scale and therefore have much greater magnitudes, of at least $10^{8} \mathrm{GeV}^{4}$. The main CC Problem is that these magnitudes of are a huge 55 orders of magnitude greater than the sum. Obviously, these two huge terms do cancel. Here we follow a phenomenological attitude and don't try solving the main CC problem. Instead we consider whether CC may vary due to IR quantum effects of massive matter fields.

It is remarkable that the same equation (17) follows from the assumption of the Appelquist and Carazzone - like decoupling for $\mathrm{CC}^{23}$. For a single particle the $\beta$-function for $\rho_{\Lambda}^{v a c}(\mu)$ is

$$
\beta_{\Lambda}^{M S}(m) \sim m^{4}
$$

hence the quadratic decoupling gives

$$
\beta_{\Lambda}^{I R}(m)=\frac{\mu^{2}}{m^{2}} \beta_{\Lambda}^{M S}(m) \sim \mu^{2} m^{2} .
$$

Then the total beta-function will be given by an algebraic sum

$$
\beta_{\Lambda}^{I R}=\sum k_{i} \mu^{2} m_{i}^{2}=\sigma M^{2} \mu^{2} \propto \frac{3 \nu}{8 \pi} M_{P}^{2} H^{2} .
$$

This leads to the same result (15), in the cosmological setting,

$$
\rho_{\Lambda}(H)=\rho_{\Lambda}\left(H_{0}\right)+\frac{3 \nu}{8 \pi} M_{p}^{2}\left(H^{2}-H_{0}^{2}\right) .
$$

It is also remarkable that one can also obtain the same $G(\mu)$, Eq. (17), in one more independent way ${ }^{13,23}$. Consider $\overline{\mathrm{MS}}$-based renormalization group equation for $G(\mu)$

$$
\mu \frac{d G^{-1}}{d \mu}=\sum_{\text {particles }} A_{i j} m_{i} m_{j}=2 \nu M_{P}^{2}, \quad G^{-1}\left(\mu_{0}\right)=G_{0}^{-1}=M_{P}^{2} .
$$

Here the coefficients $A_{i j}$ depend on the coupling constants, $m_{i}$ are masses of all particles. In particular, at one loop,

$$
\sum_{\text {particles }} A_{i j} m_{i} m_{j}=\sum_{\text {fermions }} \frac{m_{f}^{2}}{3(4 \pi)^{2}}-\sum_{\text {scalars }} \frac{m_{s}^{2}}{(4 \pi)^{2}}\left(\xi_{s}-\frac{1}{6}\right) .
$$


One can rewrite Eq. (20) as

$$
\mu \frac{d\left(G / G_{0}\right)}{d \mu}=-2 \nu\left(G / G_{0}\right)^{2} \Longrightarrow G(\mu)=\frac{G_{0}}{1+\nu \ln \left(\mu^{2} / \mu_{0}^{2}\right)} .
$$

It is easy to see that we arrived at the same formula (17), which results from covariance arguments and/or from Appelquist and Carazzone-like quadratic decoupling for the CC plus conservation law. Eq. (21) is the unique possible form of a relevant running $G(\mu)$. An alternative to this relation is the non-running, that means simply $\nu=0$.

From the perspective described above, it is not a surprise that the Eq. (21) emerges in very different approaches to renormalization group in gravity, including higher derivative quantum gravity ${ }^{26,27}$; non-perturbative quantum gravity with (hypothetic) UV-stable fixed point ${ }^{28}$ and semiclassical gravity ${ }^{29,30}$.

As far as we arrived at the two relations (15) and (16) in the cosmological setting, it is natural to construct cosmological models based on these formulas. The first steps in this directions has been done in Ref. 31 where the cosmological models with energy matter-vacuum exchange and constant $G$ were constructed in Ref. 21, where the cosmological model without matter-vacuum exchange was constructed by assuming the scale-dependence running (16) for $G$. In this presentation we will not describe the details of these models. Let us only mention that the density perturbations were explored for these models by different methods ${ }^{32,33,34,35}$. In particular, the result of Ref. 35 implies that the possible quantum contributions (17) do not really affect the power spectrum of the cosmological model, such that the last remains almost the same as in the classical case. We will discuss the importance of this result in Sect. 4.

\section{Galaxies}

If the quantum effects parametrized by Eqs. (17) really take place and are relevant even at the scale of the whole universe, they can manifest themselves also at the astrophysical scale. What could be an interpretation of $\mu$ in astrophysics?

Consider the rotation curves of galaxies. The simplest assumption is $\mu \propto 1 / r$, and this identification has been applied for the point-like model of galaxy in Refs. 36, 37 and Ref. 21. In fact, the method suggested in Ref. 21 (see also Ref. 12) is quite general, and can be used for various identifications of $\mu$. The main idea is to consider a weakly varying

$$
G=G_{0}+\delta G=G_{0}(1+\kappa) \quad|\kappa| \ll 1
$$

and perform a conformal transformation

$$
\bar{g}_{\mu \nu}=\frac{G_{0}}{G} g_{\mu \nu}=(1-\kappa) g_{\mu \nu} .
$$

It is easy to see that in the first order in $\kappa$ the metric $\bar{g}_{\mu \nu}$ satisfies usual Einstein equations with constant $G_{0}$. 
The nonrelativistic limits of the two metrics are

$$
g_{00}=-1-\frac{2 \Phi}{c^{2}} \quad \text { and } \quad \bar{g}_{00}=-1-\frac{2 \Phi_{\mathrm{Newt}}}{c^{2}} \quad,
$$

where $\Phi_{\text {Newt }}$ is the usual Newton potential and $\Phi$ is a potential of the modifies gravitational theory.

For the nonrelativistic limit of the modified gravitational force we obtain, in this way,

$$
-\Phi^{, i}=-\Phi_{\text {Newt }}^{, i}-\frac{c^{2} G^{, i}}{2 G_{0}}
$$

The formula (25) is very instructive. Quantum correction comes multiplied by $c^{2}$ and therefore it does not need to be very big to make real effect even at the galaxy scale. For a point-like model of galaxy and $\mu \propto 1 / r$ it is sufficient to have $\nu \approx 10^{-6}$ to provide the flat rotation curves ${ }^{21}$. At the same time ref. (25) shows that it not a really good choice for a non-point-like model of the galaxy. The reason is that this identification produces the "quantum-gravitational" force even if there is no mass at all.

What would be the "right" identification of the renormalization group scale parameter in the almost-Newtonian regime? Let us come back to the (QFT). Then it is clear that $\mu$ must be associated to some parameter which characterizes the energy of the particle which is transmitting gravitational interaction. Of course, $\mu \propto 1 / r$ is not the right choice.

The phenomenologically good choice is

$$
\frac{\mu}{\mu_{0}}=\left(\frac{\Phi_{\mathrm{Newt}}}{\Phi_{0}}\right)^{\alpha}
$$

where $\alpha$ is a phenomenological parameter which can be distinct for different spiral galaxies. We have found that $\alpha$ is nonlinearly growing with the mass of the galaxy.

From the QFT viewpoint the presence of $\alpha$ reflects the fact that the association of $\mu$ with $\Phi_{\text {Newt }}$ is not an ultimate choice. Remember that the vacuum EA is a relativistic object and taking $\Phi_{\text {Newt }}$ as a scale definitely ignores some relevant information. With greater mass of the galaxy the "error" in identification becomes greater too, hence we need a greater $\alpha$ to correct this. Furthermore, if $\alpha$ increase with the mass of the galaxy, it must be very small at the scale of the Sun system and of course at the scale of laboratory, when the Newton law is better verified. Finally, the recently-proposed regular scale-setting procedure gives the very same result ${ }^{38}$.

In Ref. 12 we applied the RGGR model to nine disk galaxies (including high and low surface brightness galaxies) from two sample of data ${ }^{39-40}$. We have also compared our results to three other models: a model with a dark matter halo given by the phenomenologically successful (pseudo-)isothermal profile; the Modified Newtonian Dynamics (MOND) ${ }^{41}$ (in its original form); and the Metric Skew Tensor Gravity (MSTG) ${ }^{42}$. For the shape of the rotation curve, the RGGR model has in 
general achieved lower $\chi^{2}$ and $\chi_{\text {reduced }}^{2}$ than MOND and MSTG' . Considering the expected mass to light ratio, as inferred from the Kroupa initial mass function (IMF) as derived in Ref. 39, the RGGR model has achieved better results than all the other proposals.

Since the results were very good, we are both extending our sample of disk galaxies ${ }^{15}$ and applying RGGR to elliptical galaxies ${ }^{16}$. This is important both to show that RGGR can indeed work for a larger sample and to unveil, in particular, the behavior of the $\alpha$ parameter from system to system.

Besides testing RGGR in a larger sample of disk galaxies, we are also testing the robustness of the model results once different assumptions on the baryonic matter are done. In particular, while at Ref. 12 we used exponential approximations to the matter distribution of all galaxies at all radii, we are modeling again some of the previous galaxies but using the photometric data up to the radius such data is known. An example of the newer results can be seen in Fig. 1. Comparing this result with the corresponding one in Ref. 12, it can be seen that there is no considerable difference in both the shape and the inferred parameters for this case (apart from the shape of the central region, which poses difficulties to any model ${ }^{39}$ ). For this galaxy (whose data come from Ref. 39), the inferred mass-to-light ratio and the value of $\alpha \nu$ are essentially the same of those found in Ref. $12\left(Y_{*}^{3.6} \approx 0.8\right.$ and $\alpha \nu \approx 1.7 \times 10^{-7}$, with $\left.\chi_{\text {reduced }}=1.9\right)$. In the same figure, from exactly the same data, we also show the result of applying MOND in its original form $\left(Y_{*}^{3.6} \approx 0.7 \frac{L_{\odot}}{M_{\odot}}, \chi_{\text {reduced }}=5.5\right)$. The discrepancy found from MOND directly applied to the NGC 3198 current data is a well known issue, see in particular the recent comments in Ref. 43.

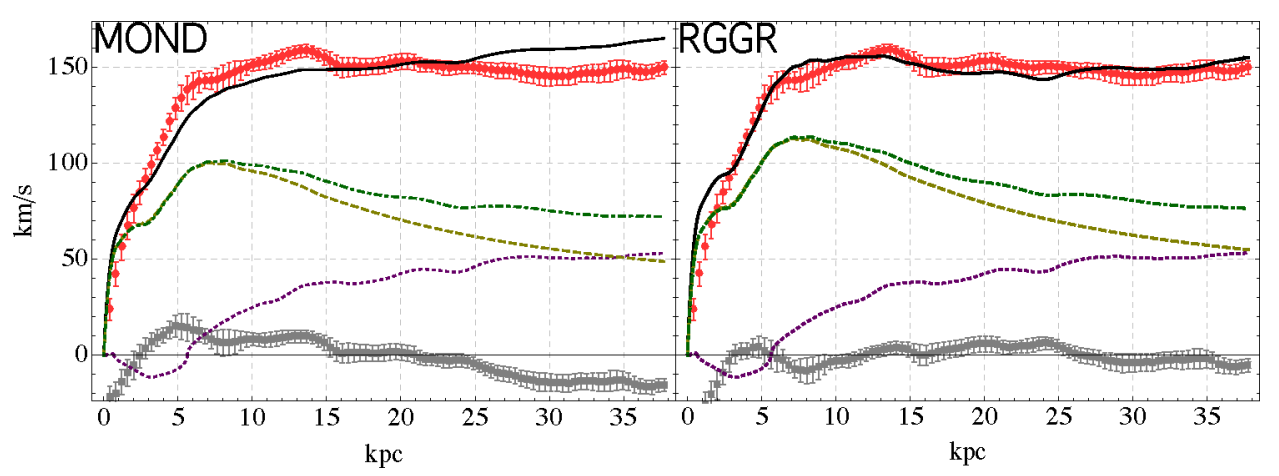

Fig. 1. NGC 3198 rotation curve fits. On the left is the fit with the original MOND prescription, and on the right the RGGR model. The upper dots and its error bars are the rotation curve observational data. The lower dots with error bars are the residues of the fit. The solid black line for each model is its best fit rotation curve, the dashed curves are the stellar rotation curves, the dotted curve is the gas rotation curve, and the dot-dashed curve is the resulting Newtonian rotation curve (both without dark matter).

\footnotetext{
${ }^{\mathrm{c}}$ The isothermal profile has one more free parameter than RGGR, while the latter has one more free parameter than MOND and MSTG.
} 


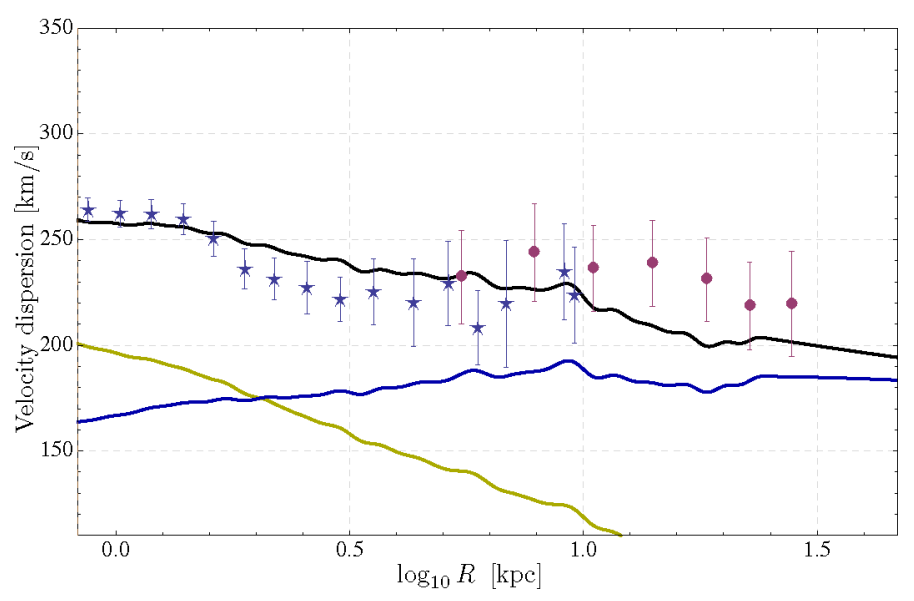

Fig. 2. RGGR mass modeling of the giant elliptical galaxy NGC 4374. The stars with the error bars are the velocity dispersion (VD) data found from the stellar photometry. The filled circles with error bars are the velocity dispersion data found from more than 450 planetary nebulae. The upper curve is the resulting best fit VD curve for the RGGR model. The descending line at the bottom is the stellar VD curve, while the crescent curve is the additional (non-Newtonian) RGGR contribution to the dispersion. The square of the last two is equal to the square of the resulting (upper) curve.

Figure 2 shows the application of RGGR to the mass modeling of the giant elliptical galaxy NGC 4374, using the same data of Ref. 44. To derive this dispersion curve, it is only necessary to replace the effective potential in the Jeans equation by the RGGR potential derived from the stellar density ${ }^{16}$. The resulting fit is very good: assuming constant anisotropy (constant $\beta$ in the Jeans equations), the RGGR model automatically leads to $\beta \approx 0.1$ (i.e., close to isotropic), to a mass-to-light ratio in perfect agreement with the Kroupa $\mathrm{IMF}^{44}, Y_{*}^{V} \approx 4 \frac{L_{\odot}}{M_{\odot}}, \nu \alpha \approx 15 \times 10^{-7}$ (in agreement with its expectation of becoming larger for larger masses) and $\chi_{\text {reduced }}=$ 1.0 .

In order to start to disclose the parameter $\alpha$ relation to the system mass, we show our partial results in the Fig. 3. The result shown in this plot is consistent with the bound $|\alpha \nu|<10^{-17}$ for the solar system, which was recently derived for the Solar system in Ref. 13 by using the weak non-conservation of the Laplace-RungeLenz vector. Further details concerning the analysis of all worked out galaxies will be available soon in Ref. 15.

\section{Cosmological applications}

It looks like we do not need CDs to explain the rotation curves of the galaxies. However, does it really mean that we can really go on with one less dark component? Maybe at the end of the day the answer will be negative, but it is definitely worthwhile to check such a possibility. 


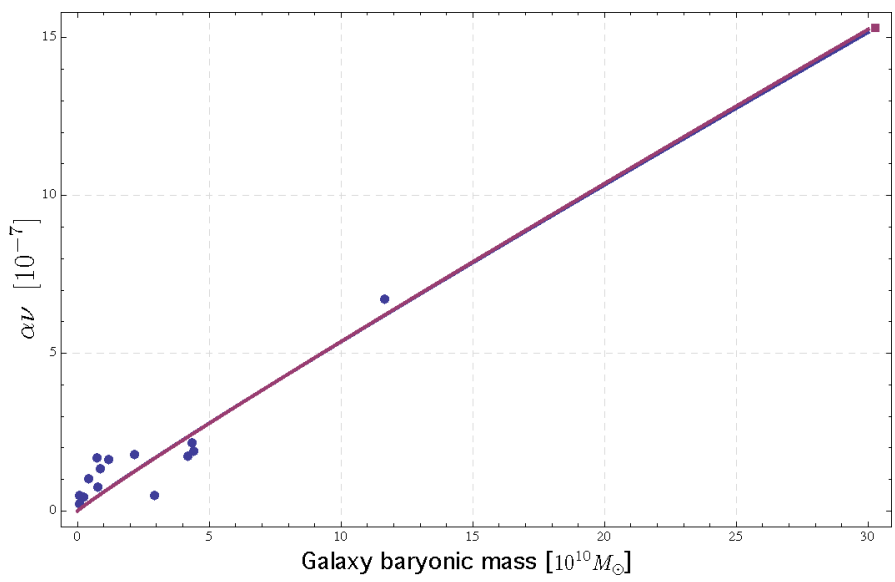

Fig. 3. A preliminary plot showing how the parameter $\alpha$ varies from galaxy to galaxy, considering their baryonic (stellar and gaseous) masses alone. Since galaxy dynamics is only sensible to $\alpha$ through the product $\alpha \nu$, in the above the total baryonic mass of 15 galaxies are plotted against $\alpha \nu ; 14$ of them are disk galaxies (depicted by circles), and one is a giant elliptical galaxy (NGC 4374), depicted by a square. There are two lines in the above plot that are almost identical. Both were derived from the best fit of $\alpha=a \operatorname{Mass}^{b}$, where $a$ and $b$ are constants, but one of them considering only the disk galaxies and the other all the 15 galaxies. For this range of masses, $b \approx 1$ is a solution of the fit, regardless whether the elliptical NGC 4374 is part of the data or not.

It is well known that the main requests for the DM come from the fitting of the LSS, CMB, BAO, lensing etc. However there is certain hope to replace, e.g., $\Lambda \mathrm{CDM}$ by a WDM, e.g., by sterile neutrino with much smaller $\Omega_{D M}$. So, the idea it to trade the set of $\left(\Omega_{B M}, \Omega_{D M}, \Omega_{C C}\right)$ from the conventional $(0.04,0.23,0.73)$ to $(0.04,0.0 x, 0.9(1-x))$ with a relatively small $x$. Such a new concordance model would have less relevant coincidence problem, and in general such a possibility is interesting to verify. The first move in this direction has been done recently in Ref. 14 by using the Reduced Relativistic Gas (RRG) model.

The RRG model is a Simple cosmological model of a universe filled by ideal relativistic gas of massive particles ${ }^{45}$. As an approximation we assume that all of these particles have the same kinetic energy. The Equation of State (EOS) of such gas is ${ }^{45,46}$

$$
P=\frac{\rho}{3}\left[1-\left(\frac{m c^{2}}{\varepsilon}\right)\right]^{2}=\frac{\rho}{3}\left(1-\frac{\rho_{d}^{2}}{\rho^{2}}\right) .
$$

In this formula $\varepsilon$ is the kinetic energy of the individual particle, $\varepsilon=m c^{2} / \sqrt{1-\beta^{2}}$. Furthermore, $\rho_{d}=\rho_{d 0}^{2}(1+z)^{3}$ is the mass (static energy) density. One can use one or another form of the equation of state (27), depending on the situation. The nice thing is that one can solve the Friedmann equation in this model analytically. The deviation from Maxwell or relativistic Fermi-Dirac distribution is less than $2.5 \%$. It 
is amusing that the same EOS has been used in Ref. 46 by A.D. Sakharov in 1965 to predict the oscillations in the CMB spectrum for the first time.

In Ref. 14 we have used RRG without quantum effects to fit such sets of observational data as Supernova type Ia (Union2 sample), $H(z)$, CMB ( $R$ factor), BAO and LSS (2dfGRS). Taking all these tests together we confirm that the $\Lambda$ CDM is definitely the most favored model. As far as we tried the model without quantum effects, this output can be seen as a successful test of RRG and nothing else.

However, there is a very important extra detail which concerns the LSS part alone. In this case we met the possibility of an alternative model with a small quantity of a WDM. This output is potentially relevant in view of the fact that (as we have already emphasized above) the LSS is the only test which can not be affected by the possible quantum renormalization-group running in the low-energy gravitational action. Let us present here a few details of the results of Ref. 14 .

The cosmological model based on RRG with the presence of the cosmological constant admits an analytic solution for the energy density. This solution does interpolates between the radiation-dominated and the matter-dominated $\operatorname{eras}^{45,46}$. It can represent a warm dark matter(WDM), characterized by the parameter, $b=$ $\frac{\beta}{\sqrt{1+\beta^{2}}}$.

In Ref. 47 the model was successfully used to make a analysis of density perturbations and comparison with the $2 \mathrm{dFGRS}$ data. Using the RRG model to derive and analyze density perturbations at the linear level one arrives at the conclusion
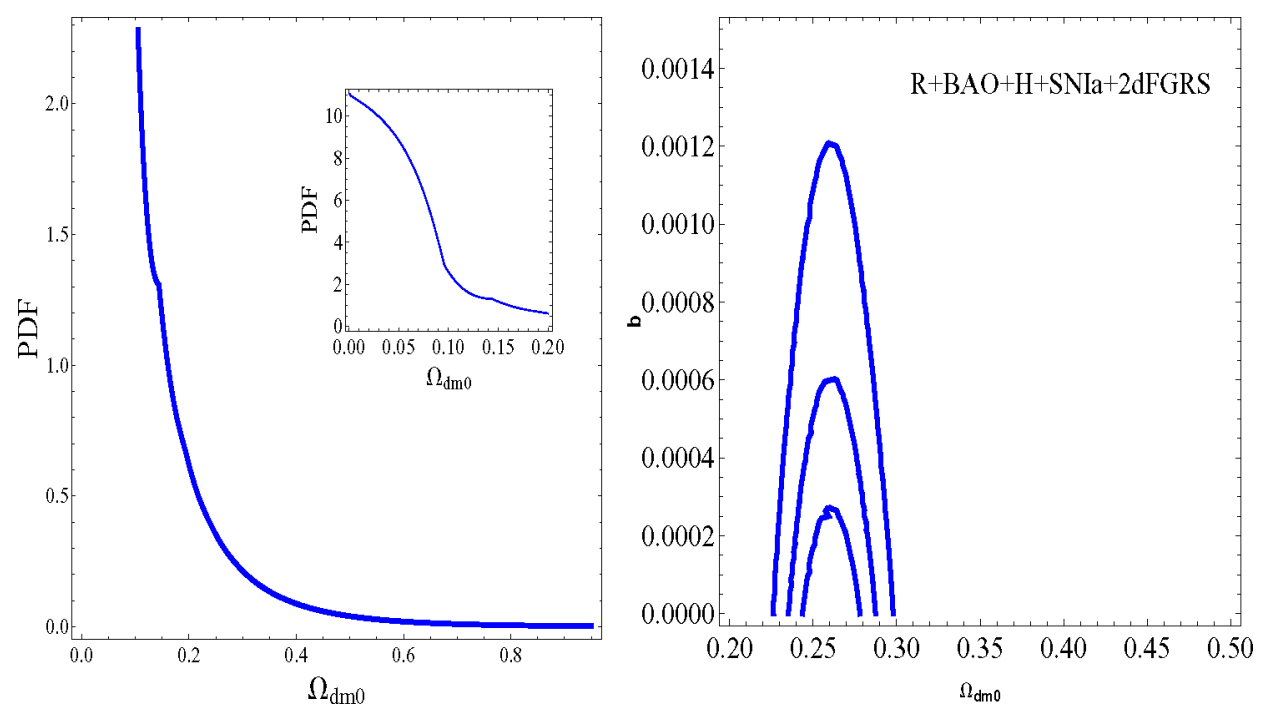

Fig. 4. The probability density function using 2 dFGRS for $\Omega_{m 0}$ (left). To build the PDF we marginalize the free parameters considering the intervals: $\Omega_{\mathrm{dm} 0} \epsilon[0.05,0.95]$ and $b \epsilon[0.001,0.4]$, for each case. As we can see the model is included,i.e, the first figure shows that for a $\Omega_{\mathrm{dm} 0} \approx 0.25$ corresponds to a $b \approx 0$. Confidence regions at $1-\sigma, 2-\sigma$ and $3-\sigma$ levels(right) from inner to outer respectively on the $\left(\Omega_{\mathrm{dm} 0}, b\right)$ plane for our relativistic model in the flat case. 
that the upper bound for the warmness parameter is $b \leq 3-4 \times 10^{-5}$. It is about two order of magnitude smaller than the escape velocities for the spiral galaxies. This result was similar to those obtained using non-analytical models of WDM based on the system of Boltzmann-Einstein equations.

As we have already mentioned, in Ref. 14 the model has been tested using four Supernova type Ia (Union2 sample), $H(z), \mathrm{CMB}$ ( $R$ factor) and BAO. Moreover, a detailed study of structure formation at linear level has been performed using the 2dFGRS data for matter power spectrum. The different tests have been crossed in order to obtain a more clear evaluation for the free parameters, which are essentially the velocity parameter for the dark matter particles $b$ and the dark matter ratio to the critical density $\Omega_{\mathrm{dm} 0}$. All the analysis has been performed using the flat universe prior. In general, we confirm that $\Lambda$ CDM is the most favored model. However, for the LSS data the maximum probability for $\Omega_{\mathrm{dm} 0}$ occurs at a zero value. This seems to be a consequence of the restriction of the analysis to a linear level, since a certain amount of dark matter is necessary in order to have the formation of structure process. In any case, a small amount of dark matter is certainly admitted much less than that predicted by the $\Lambda$ CDM model. The results are shown in Fig. 1. Therefore, for the 2dFGRS data alone we met the possibility of an alternative model with a small quantity of a WDM. This output is potentially relevant in view of the fact that the LSS is the only test which can not be affected by the possible quantum renormalization-group running in the low-energy gravitational action.

\section{Conclusions and discussions}

The evaluation of quantum corrections from massive fields is, to some extent, reduced to existing-nonexisting paradigm. There is no theoretical way to prove or disprove the existence of such quantum corrections ${ }^{10}$ and on has to rely on faith or use phenomenological approach, that means simply assume the existence of such quantum corrections and check their possible consequences. In this way we arrive at the cosmological and astrophysical model with one free parameter plus certain freedom of scale identification. It turns out that the rotation curves of all tested galaxies can be described by the $G(\mu)$ formula. The situation with clusters and other tests, especially $\mathrm{CMB}$ and gravitational lensing, remains unclear, because it was not explored at all. At the same time, we have a very strong positive signal from the analysis of the LSS data. The power spectrum tests are almost not sensible to the $G(\mu)$ running and, exactly in this case, we meet an alternative to $\Lambda$ CDM in the zero-order approximation ${ }^{14}$.

Finally, we can conclude that there is still some (albeit they can be evaluated to be small) chance that the vacuum effects of QFT in an external gravitational field play more significant role in our Universe that we usually think. In particular, we gain a chance to resolve the so-called coincidence problem for the $\mathrm{CC}$ in a qualitatively new way. This problem consists in the question of why our Universe is 
such that the cosmic acceleration has started only recently. However, if the presentday $\Omega_{C C}$ is more than 0.9 , the moment when this acceleration starts move essentially back to the past and there is no such question. Of course, many tests of the possible cosmic-scale effects of quantum corrections are necessary before one can think about this solution seriously, but the results of preliminary studies described here indicate that the subject is interesting and it is worthwhile to study it in more details, from both phenomenological and theoretical sides.

\section{Acknowledgments}

I.Sh. is very grateful to the Organizers of the QFEXT-2011 meeting in Benasque for invitation and for the possibility to present our work as a plenary talk. We thank W.J.G. de Blok and N.R. Napolitano for providing us observational data on the galaxies NGC 3198 and NGC 4374. The work of I.Sh. has been partially supported by CNPq, FAPEMIG and ICTP. J.F. was partially supported by CNPq.

\section{References}

1. R. J. Riegert, Phys. Lett. B 134, 56 (1984).

2. E.S. Fradkin and A.A. Tseytlin, Phys. Lett. B 134, 187 (1984).

3. I.L. Shapiro, Local conformal symmetry and its fate at quantum level, [hepth/0610168]; PoS IC2006:030,2006.

4. M.J. Duff, Class. Quant. Grav. 11, 1387 (1994).

5. R. Balbinot, A. Fabbri and I.L. Shapiro, Phys. Rev. Lett. 83, 1494 (1999); Nucl. Phys. B559, 301 (1999).

6. P.R. Anderson, E. Mottola, R. Vaulin, Phys. Rev. D76, 124028 (2007).

7. A.A. Starobinski, Phys. Lett. 91B, 99 (1980); Nonsingular Model of the Universe with the Quantum-Gravitational De Sitter Stage and its Observational Consequences, Proceedings of the second seminar "Quantum Gravity", pp. 58-72 (Moscow, 1982); JETP Lett. 30, 682 (1979); 34, 438 (1981); Sov. Astron. Lett. 9, 302 (1983).

8. A.M. Pelinson, I.L. Shapiro and F.I. Takakura, Nucl. Phys. B648, 417 (2003).

9. S. Deser and A. Schwimmer, Phys. Lett. 309B, 279 (1993).

S. Deser, Phys. Lett. 479B, 315 (2000).

10. I. L. Shapiro, J. Solà, Phys. Lett. B682, 105 (2009).

11. I.L. Shapiro, J. Solà, Phys. Lett. B530, 10 (2002).

12. D. C. Rodrigues, P. S. Letelier, and I. L. Shapiro. JCAP 1004, 020 (2010); see also arXiv:1102.2188 [astro-ph.CO; gr-qc].

13. C. Farina, W. J. M. Kort-Kamp, S. Mauro, I.L. Shapiro, Phys. Rev. D83, 124037 (2011).

14. J.C. Fabris, I.L. Shapiro and A.M. Velasquez-Toribio, Phys. Rev. D85, 023506 (2012).

15. P. L. de Oliveira, D. C. Rodrigues, J. Fabris, I. L. Shapiro. work in progress.

16. D. C. Rodrigues, Elliptical galaxies kinematics within general relativity with renormalization group effects, arXive: 1203.2286.

17. T. Appelquist and J. Carazzone, Phys. Rev. 11D, 2856 (1975).

18. E.V. Gorbar, I.L. Shapiro, JHEP 02, 021 (2003).

19. E.V. Gorbar, I.L. Shapiro, JHEP 06, 004 (2003).

20. I.L. Shapiro, Class. Quantum Grav. 25, 103001 (2008).

21. I.L. Shapiro, J. Solà, H. Stefancic, JCAP 0501, 012 (2005). 
22. A. Babic, B. Guberina, R. Horvat, H. Štefančić, Phys. Rev. D71, 124041 (2005).

23. I.L. Shapiro, J. Solà, JHEP 02, 006 (2002).

24. J. Solà, J. Phys. A41 164066 (2008); hep-th/0710.4151. (Contribution to the Proceeding of QFEXT-08 meeting, Leipzig - 2007).

25. S. Weinberg, Rev. Mod. Phys. 61, 1 (1989).

26. A. Salam and J. Strathdee, Phys. Rev. D18, 4480 (1978).

27. E.S. Fradkin and A.A. Tseytlin, Nucl. Phys. 201B, 469 (1982).

28. A. Bonanno and M. Reuter, Phys. Lett. B527, 9 (2002); Phys. Rev. D65, 043508 (2002).

29. B.L. Nelson and P. Panangaden, Phys. Rev. D25, 1019 (1982).

30. I.L. Buchbinder, S.D. Odintsov and I.L. Shapiro, Effective Action in Quantum Gravity (IOP Publishing, Bristol, 1992).

31. I.L. Shapiro, J. Solà, C. España-Bonet, P. Ruiz-Lapuente, Phys. Lett. 574B, 149 (2003); JCAP 0402, 006 (2004);

I.L. Shapiro, J. Solà, Nucl. Phys. Proc. Suppl. 127, 71 (2004).

32. R. Opher, A. Pelinson, Phys. Rev. D70, 063529 (2004), astro-ph/0405430.

33. P. Wang, X.H. Meng, Class. Quant. Grav. 22, 283 (2005).

34. J.C. Fabris, I.L. Shapiro and J. Solà, JCAP 0702, 016 (2007);

A.M. Velasquez-Toribio, arXiv:0907.3518 [astro-ph.CO].

35. J. Grande, J. Solà, J.C. Fabris and I.L. Shapiro, Class. Quant. Grav. 27, 105004 (2010).

36. J.T.Goldman, J.Perez-Mercader, F.Cooper and M.M.Nieto, Phys. Lett. B281, 219 (1992);

O. Bertolami, J.M. Mourao \& J. Perez-Mercader, Phys. Lett. B311, 27 (1992).

37. M. Reuter \& H. Weyer, Phys. Rev. D70, 124028 (2004); JCAP 0412, 001 (2004).

38. S. Domazet, H.Štefančić, Phys. Lett. B703, 1 (2011).

39. W. J. G. de Blok, F. Walter, E. Brinks, C. Trachternach, S. Oh, and R. C. Kennicutt. High-Resolution Rotation Curves and Galaxy Mass Models from THINGS. Astron. J., 136:2648-2719, Dec. 2008.

40. G. Gentile, P. Salucci, U. Klein, D. Vergani, and P. Kalberla. The cored distribution of dark matter in spiral galaxies. Mon. Not. Roy. Astron. Soc., 351:903, 2004.

41. M. Milgrom. A modification of the Newtonian dynamics - Implications for galaxies. Astrophys. J., 270:371-389, July 1983.

42. J. R. Brownstein and J. W. Moffat. Galaxy Rotation Curves Without Non-Baryonic Dark Matter. Astrophys. J. 636721 (2006).

43. G. Gentile, B. Famaey, and W. J. G. de Blok. THINGS about MOND. 2010.

44. N. R. Napolitano, A. J. Romanowsky, M. Capaccioli, N. G. Douglas, M. Arnaboldi, L. Coccato, O. Gerhard, K. Kuijken, M. R. Merrifield, S. P. Bamford, A. Cortesi, P. Das, and K. C. Freeman. The PN.S Elliptical Galaxy Survey: a standard $\Lambda$ CDM halo around NGC 4374? Mon. Not. Roy. Astron. Soc., 411:2035-2053, Mar. 2011.

45. G. de Berredo-Peixoto, I. L. Shapiro and F. Sobreira, Mod. Phys. Lett. A20, 2723 (2005).

46. A.D. Sakharov, Soviet Physics (JETP), 49, 345 (1965).

47. J.C. Fabris, I.L. Shapiro and F. Sobreira, JCAP 0902, 001 (2209). 